\title{
Shape Analysis of Segmentation Variability
}

\author{
Jess D Tate ${ }^{1}$, Nejib Zemzemi ${ }^{2}$, Wilson W Good ${ }^{1}$, Peter van Dam ${ }^{3}$, \\ Dana H Brooks ${ }^{4}$, and Rob S MacLeod ${ }^{1}$ \\ ${ }^{1}$ University of Utah, Salt Lake City, Utah, USA \\ ${ }^{2}$ Inria Bordeaux Sud Ouest, France \\ ${ }^{3}$ UMC Utrecht, The Netherlands \\ ${ }^{4}$ Northeastern University, Boston, MA, USA
}

\begin{abstract}
Patient-specific cardiac simulation rely on accurate geometric models extracted from medical images. Segmentation of cardiac images is a key, yet possibly error-prone part of patient-specific simulations, e.g., heart propagation models, ECG forward simulation, and ECG Imaging. In this study, we performed shape analysis on multiple segmentations of the same patient to quantify variability. We found that segmentation shape varied most in the basal region of the ventricles and the right ventricular outflow tract in all three structures, which could have significant impact on pipelines that depend on geometric models. The statistical shape-model generated using ShapeWorks provides a pathway to subsequently quantify the impact of the segmentation variability on modeling pipelines with uncertainty quantification.
\end{abstract}

\section{Introduction}

Patient-specific cardiac simulation is becoming increasingly relevant as a research and clinical tool for predicting arrhythmias and guiding treatments. These simulation methods, such as heart propagation models [1], ECG forward simulation, and Electrocardiographic Imaging (ECGI)[2,3], rely on interpreting clinical data, especially medical images, into computer models. Although many of these pipelines are continually improving and emerging into clinical use, variability from the interpretation of clinical inputs remains largely unquantified for many approaches.

Many assumptions and estimations comprise the interpretation of clinical data into a tractable cardiac models, leading to multiple possible sources of uncertainty. One [such source, often overlooked, is the segmentation of the geometric model, particularly the heart, because it usually requires manual input and user judgements. We have previously shown that segmentations of the same patient ge- ometry can vary widely, especially the cardiac surface [4] and this variability likely affects the ECGI solutions [5]. However, the field still lacks a sufficiently quantifiable description of the variability of segmentation shape and its resulting affects on cardiac simulations.

In this study, we quantified segmentation shape variability and generated a statistical shape-model to facilitate uncertainty quantification of cardiac modeling pipelines. We used the collaborative framework of the Consortium for ECG Imaging (CEI) to generate multiple segmentations of the same patient to provide sufficient data for computing shape variability of cardiac segmentations, and used a correspondence-based shape analysis to calculate shape statistics [and-in] a parameterized shape space. We found the areas of segmentation variability agreed, generally, with our previous analysis of ECGI variability [5], and that the shape space could provide a parameterized geometric model for use in uncertainty quantification.

\section{Methods}

To quantify variability of cardiac segmentation, we used statistical shape analysis on multiple ventricular segmentations. A single patient CT scan was segmented by nine researchers within the CEI and shape analysis was applied using ShapeWorks [6] (https://www.sci.utah.edu/software/shapeworks.html). ShapeWorks performs shape analysis by finding correspondence points across all input geometries using a particle system optimizer. The optimization attempts to maximize distance between points while minimizing the number of modes of variation across the cohort of geometries. The correspondence points, shown in Figure 1, facilitate regional comparison of the geometries and subsequent shape analysis. The original ventricular segmentations did not produce stable correspondence solutions in ShapeWorks, likely due to the concave shape of the ventricles, so each was split into three separate surfaces: epicardium, left ventricular (LV) endocardium, and right ventricular (RV) en- 


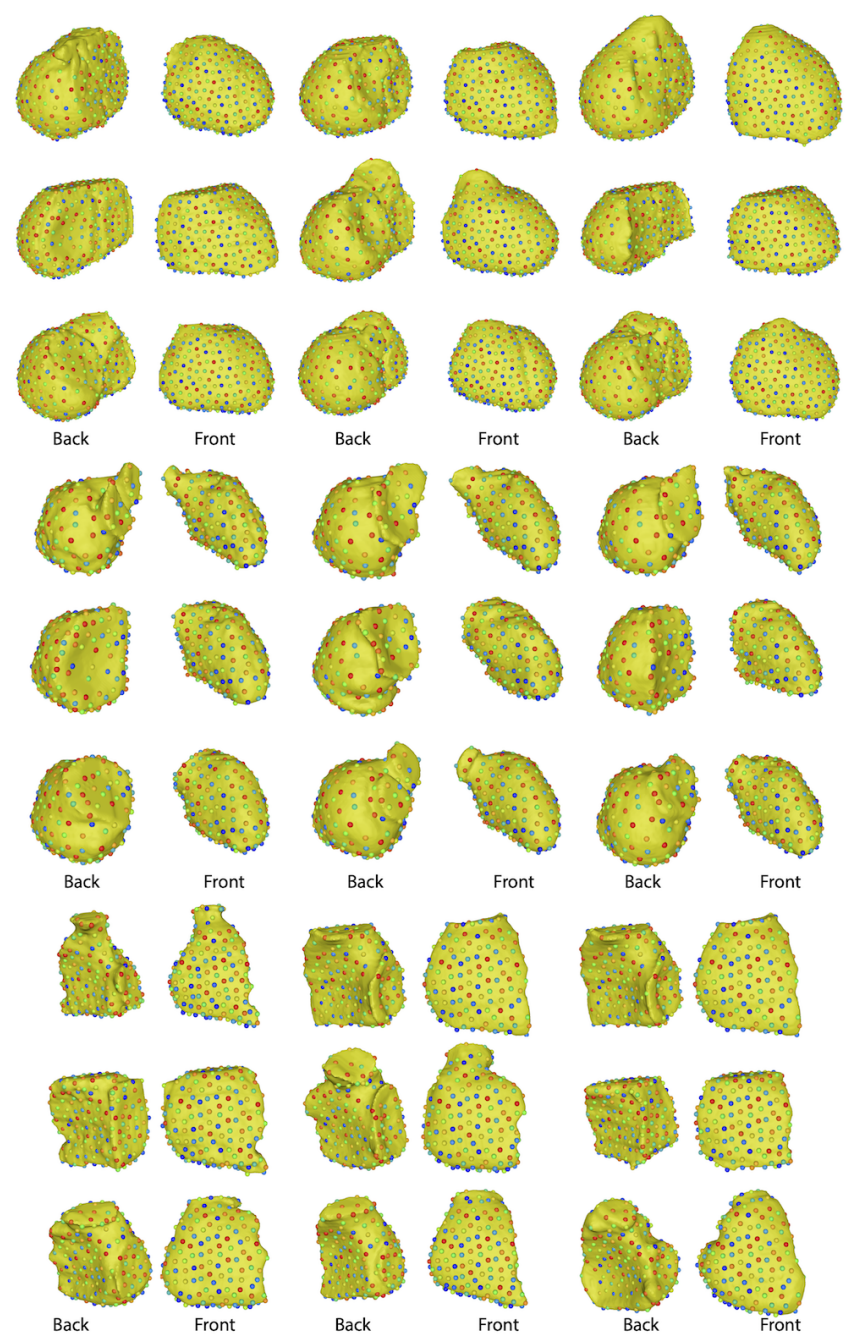

Figure 1. Correspondence points generated by ShapeWorks for all segmentations.

docardium. This produced three cohorts with correspondence points for shape analysis.

The shape analysis performed in this study included Euclidean distance statistics and a principle component analysis (PCA) to determine modes of shape variation. For each of the three set of surfaces, a mean geometry was created from the computed correspondences. The standard deviation of the Euclidean distance from each correspondence point to the mean geometry was calculated to create a map of local shape variability. PCA was also performed on the correspondence point sets, identifying seven or eight orthogonal axes, or modes, of variation for each of the 3 surface sets. We limited analysis to the first four modes of variation, which encompassed $89.3 \%, 87.2 \%$, and $89.2 \%$ of the total variability for the epicardium, $\mathrm{LV}$ endocardium, and RV endocardium, respectively. For the four major modes of variation, we computed the standard deviation of

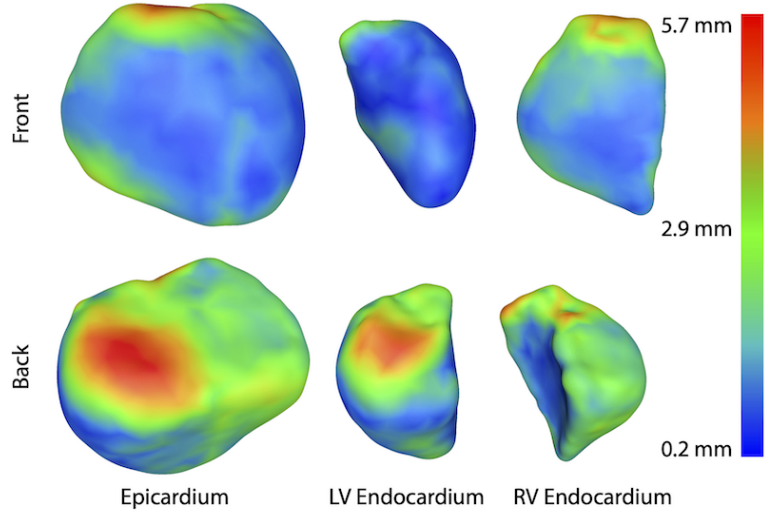

Figure 2. Local cohort variability computed by the standard deviation of the euclidian distance of each point set to the mean point set.

the distribution and generated geometries at intervals along the principle axes. The axes of variation and the distribution along each axis constitute the statistical shape-model.

The medical images used in this study were collected by Sapp et al. [7] and are available for open use on the EDGAR database (http://edgar.sci.utah.edu) [8] a shared resource of the CEI.

\section{Results}

Figure 1 demonstrates the results of the particle system optimization, i.e., the computed correspondence points. Qualitatively, the points are generally evenly spaced and cover the macro structures of each of the surfaces. In some submissions, there were regions, such as the RV apex and RV outflow tract (RVOT) that had relatively sparser sampling than the rest of the surface. However, the coverage of the correspondences generated was sufficient to allow for suitable shape analysis.

Shape analysis of the segmentations showed regions of high variability across the three surfaces. Figure 2 shows high variability in the Euclidean distances of the point sets in regions along the base and the RVOT. Other areas of variability include the posterior wall and the RV apex. Figure 4 shows high qualitative agreement between the local variability along the primary mode of variation and the euclidean distance variation (Figure 2), albeit with a higher amplitude. Furthermore, Figure 4 shows that other modes of variation contain the most variability in the same locations as the primary mode, yet the relative variability among those regions shift. For instance, while the RVOT demonstrates high variability in all modes, the area of highest standard deviation can occur at different locations along the annulus of the pulmonary valve: the whole annulus in axis 0 , the center and the endocardium in axis 1 , on the base in axes 2 and 3, and on the anterior annulus 

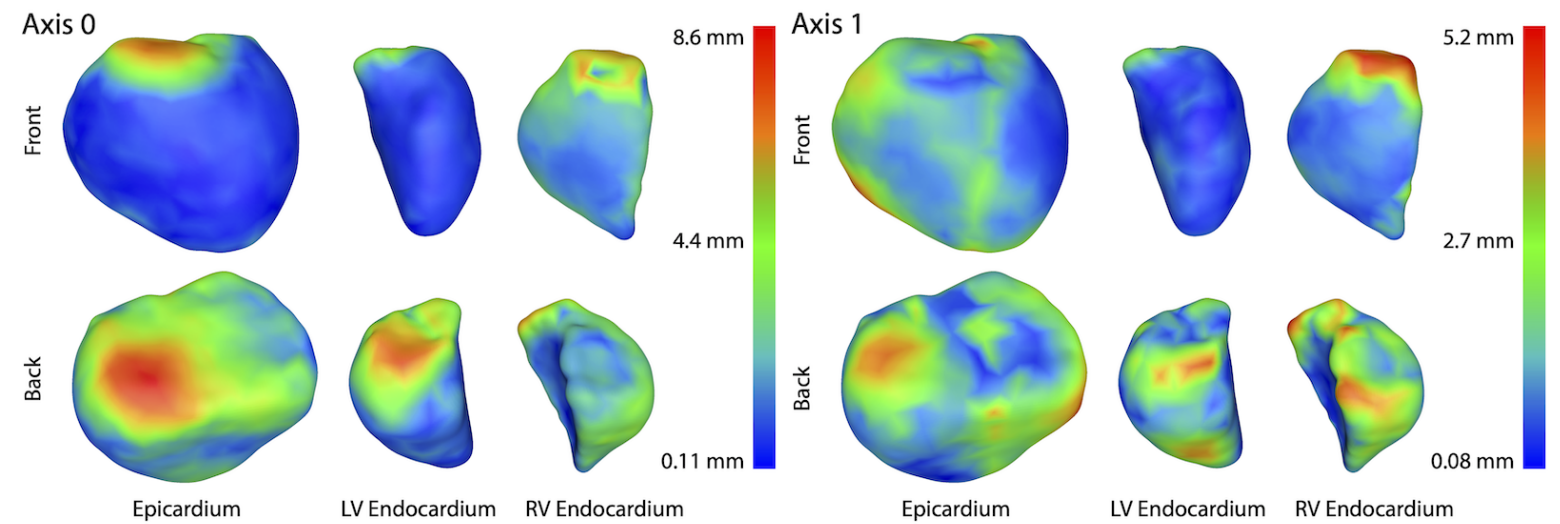

Axis 2
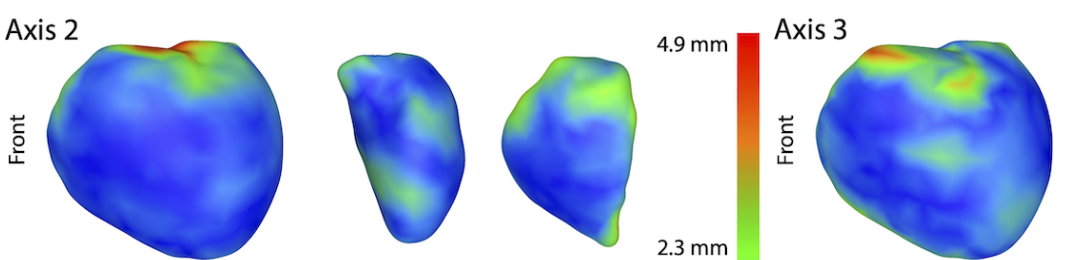

LV Endocardium

RV Endocardium
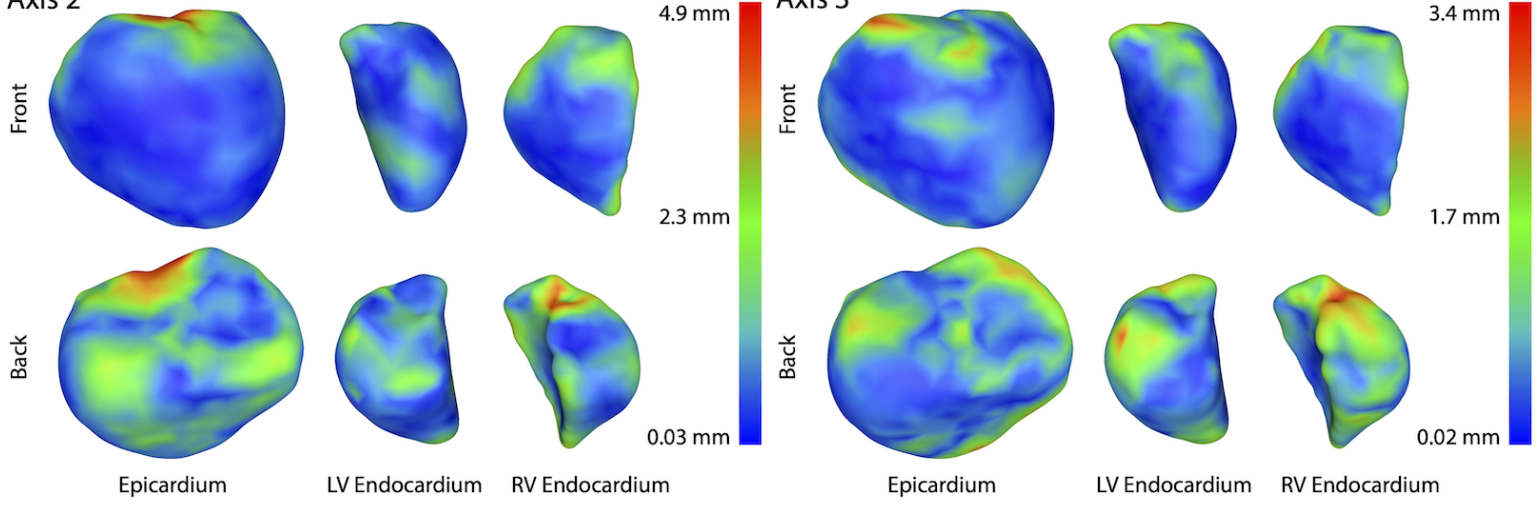

Epicardium

LV Endocardium RV Endocardium

Figure 3. Local standard deviation along the 4 major modes of variation.

in axis 3. Figure 4 shows the geometric changes that occur along each of the principle axes. The standard deviations in shape space along each of the axis of variation are 63.8, 42.7, 29.2, and $20.8 \mathrm{~mm}$, respectively, for the epicardium; 35.6, 23.0, 15.1, and $13.5 \mathrm{~mm}$ for the $\mathrm{LV}$ endocardium; and $42.1,31.2,22.5$, and 17.2 for the RV endocardium.

\section{Discussion and Conclusions}

The results in this study indicate substantial variability in regions of the cardiac segmentation that may affect the results of cardiac simulations. Additionally, the results of the study involves generating a parameterized shape-model that can be used in cardiac modeling pipelines to compute uncertainty.

The regions of high variability shown near the base and RVOT (Figures $2 \& 3$ ) matches results found in our previous work that analyzed segmentation based on closest distance to an aggregate segmentation generated with STAPLE [5]. These regions aligned with high variability of the ECGI solution, suggesting a dependence of simulation results on segmented shape variability. However, our current shape analysis also identified regions of variability on the posterior wall and near the apex, areas which previously showed less variability in the ECGI solution [5]. However, more analysis is needed to explore the link between the high cardiac segmentation variability simulation results.

The shape analysis performed with ShapeWorks includes generating a shape space from the PCA which acts as a statistical shape-model. Any geometry within the shape space can be recreated by a linear combination of the modes of variation. Additionally, the projection of the cohort geometries onto each of the axes can be described with a scalar distribution. The mean geometries, axes of variation, each defined as a set of $3 \mathrm{D}$ vectors for each point, and the distributions on each axis can be used as the inputs of uncertainty tools such as polynomial chaos [9] to provide a estimation of the uncertainty of simulations due to the segmentation variability.

While it is a relatively small part of many cardiac modeling pipelines, segmentation of the heart is an uncertain and impactful aspect of simulation. The results of this study will lead to better quantification of these sensitivities by combining shape-models with uncertainty tools like UncertainSCI (https://github.com/SCIInstitute/UncertainSCI).

\section{Acknowledgments}

This project was supported by the National Institute of General Medical Sciences of the National Insti- 

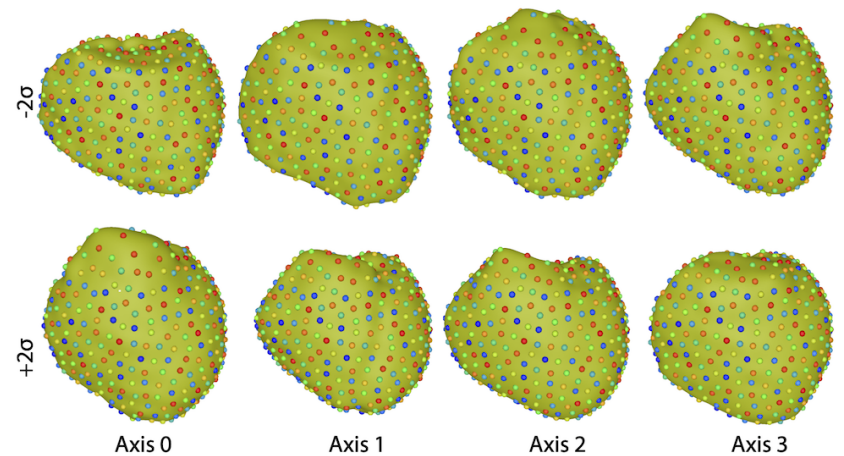

$\stackrel{\text { Tे }}{1}$
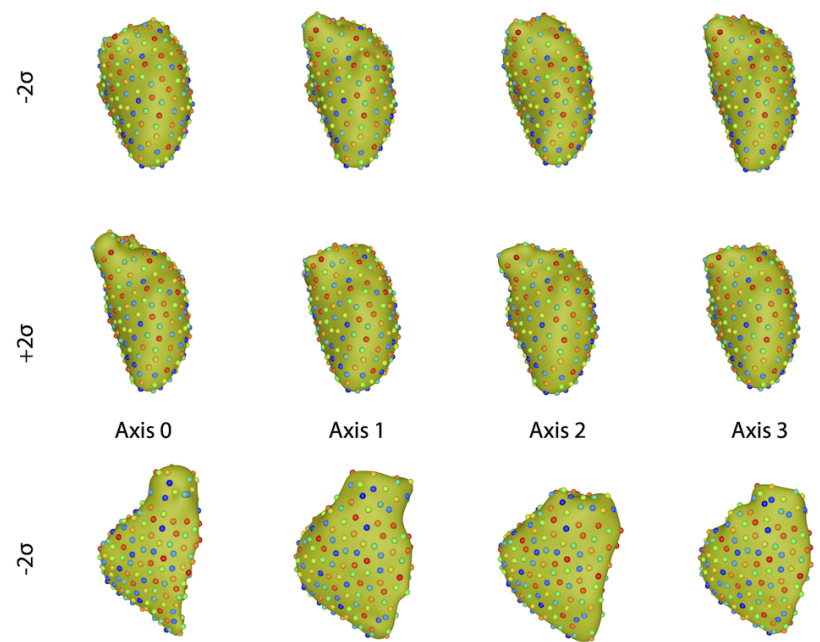

Axis 2

Axis 3

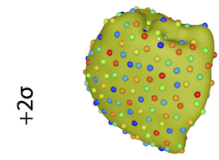

Axis 0

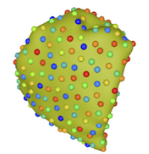

Axis 1
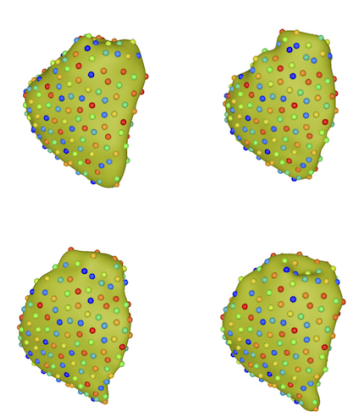

Axis 2

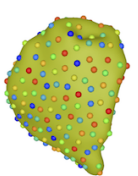

Axis 3

Figure 4. Shape changes along the four major modes of variation at two standard deviations $(\sigma)$.

tutes of Health under grant numbers P41GM103545, R24GM136986, U24EB029012, U24EB029011, R01AR076120, and R01HL135568. Data used in this study was made available by Drs. John Sapp and Milan Horáček and their research collaboration with Dalhousie University. Thanks to Sophie Giffard-Roisin, Eric Perez-Alday, Laura Bear, Beáta Ondrušová, Jana Svehlikova, Machteld Boonstra, Martim Kastelein, and Maryam Tolou for providing segmentations for this study.

\section{References}

[1] Vigmond E, Hughes M, Plank G, Leon L. Computational tools for modeling electrical activity in cardiac tissue. J Electrocardiol 2003;36 Suppl:69-74.

[2] Barr R, Ramsey M, Spach M. Relating epicardial to body surface potential distributions by means of transfer coefficients based on geometry measurements. IEEE Trans Biomed Eng Jan. 1977;24:1-11.

[3] Gulrajani R. The forward and inverse problems of electrocardiography. EMBS Mag Sep./Oct. 1998;17(5):84-101.

[4] Ghimire S, Dhamala J, Coll-Font J, Tate JD, Guillem MS, Brooks BH, MacLeod RS, Wang L. Overcoming barriers to quantification and comparison of electrocardiographic imaging methods: A community- based approach. In Computing in Cardiology Conference (CinC), 2017, volume 44. 2017; $1-4$.

[5] Tate JD, Zemzemi N, Good WW, van Dam P, Brooks DH, MacLeod RS. Effect of segmentation variation on ECG imaging. In Computing in Cardiology, volume 45. September 2018; .

[6] Cates J, Meyer M, Fletcher P, Witaker R. Entropy-based particle systems for shape correspondence. In Workshop on Mathematical Foundations of Computational Anatomy, MICCAI 2006. October 2006; 90-99. URL http://www.sci.utah.edu/publications/ cates06/Cates-miccai2006.pdf.

[7] Sapp JL, Dawoud F, Clements JC, Horáček BM. Inverse solution mapping of epicardial potentials: Quantitative comparison with epicardial contact mapping. Circ Arrhythm Electrophysiol October 2012;5(5):1001-1009. ISSN 19413149. URL http://circep.ahajournals.org/ content/5/5/1001.

[8] Aras K, Good W, Tate J, Burton B, Brooks D, Coll-Font J, Doessel O, Schulze W, Patyogaylo D, Wang L, van Dam P, MacLeod R. Experimental data and geometric analysis repository: EDGAR. J Electrocardiol 2015;48(6):975-981.

[9] Swenson D, Geneser S, Stinstra J, Kirby R, MacLeod R. Cardiac position sensitivity study in the electrocardiographic forward problem using stochastic collocation and BEM. Annal Biomed Eng Dec. 2011;30(12):2900-2910.

Address for correspondence:

Jess Tate

Scientific Computing and Imaging Institute

WEB, 72 South Campus Drive, Rm 3750

Salt Lake City, UT 84112

jess@sci.utah.edu 